\title{
Symposium
}

\section{Portal hypertension}

\author{
Brig J.K. Banerjee \\ Consultant, Department of Surgery \& GI Surgery, Command Hospital (CC), Lucknow 226002, India
}

\section{Introduction}

The subject of portal hypertension has been dealt in detail in medical literature, however majority of data is from Western countries where the etiology, scope of management and outcome is very different from our patients, or the patients seen in under developed tropical countries. It is therefore necessary to look at PHT with this perspective.

\section{Definition}

Portal hypertension (PHT) indicates increased pressure in portal venous system. Normal portal venous pressure is $10 \mathrm{mmHg}\left(14 \mathrm{~cm}\right.$ of $\left.\mathrm{H}_{2} \mathrm{O}\right) \cdot{ }^{1}$ Patients usually present with splenomegaly, life threatening gastrointestinal bleeding and ascites. Due to a large variation in the common etiologies of PHT and availability of emergency medical facilities for patients in Western countries vis-a-vis patients in tropical countries, the presentation, management and outcome differs significantly. ${ }^{2}$

\section{Etiology and pathophysiology}

Portal venous system drains blood from intestine, spleen and pancreas into the liver mainly via the superior mesenteric, inferior mesenteric and splenic veins. The splenic vein and superior mesenteric vein join behind the head of pancreas to continue as portal vein in the free margin of hepatoduodenal ligament. Near the hilum of liver, portal vein divides into right and left branches that supply the right and left hepatic lobes respectively. The portal vein supplies liver with $80 \%$ of its blood and $20 \%$ of its oxygen requirement. It also carries an anti atrophic factor which prevents atrophy of hepatic cells. Since the portal venous system is a valveless system, pressure anywhere in the system is same. The pressure in portal venous system can rise either due to an obstruction in the extra hepatic portal venous system, or due to increase in resistance to portal blood flow. This resistance to blood flow can occur commonly at the level of sinusoids or prior to that. Based on this the PHT is classified as under:

\begin{tabular}{|c|c|c|c|}
\hline $\begin{array}{l}\text { Srl. } \\
\text { No. }\end{array}$ & Classification & Site of block & Example \\
\hline 1. & Pre hepatic & Pre sinusoidal & $\begin{array}{l}\text { Extra hepatic portal } \\
\text { venous obstruction } \\
\text { (EHPVO) }\end{array}$ \\
\hline 2. & Intrahepatic & b. Sinusoidal & $\begin{array}{l}\text { Non cirrhotic portal } \\
\text { fibrosis (NCPF) } \\
\text { Schistosomiasis } \\
\text { Cirrhosis }\end{array}$ \\
\hline 3. & Post hepatic & Post sinusoidal & $\begin{array}{l}\text { Hepatic venous outflow } \\
\text { tract obstruction (HVOTO) } \\
\text { Budd Chiari syndrome } \\
\text { Constrictive pericarditis }\end{array}$ \\
\hline
\end{tabular}

Relative incidence of different etiologies of PHT in India vis a vis Western countries is quite different. ${ }^{1}$

\begin{tabular}{lll} 
Etiology & India & West \\
\hline Cirrhosis & $<50 \%$ & $>80 \%$ \\
NCPF & $10-25 \%$ & $3-5 \%$ \\
EHPVO & $30-40 \%$ & $5 \%$ \\
Budd - Chiari Syndrome & $8-26 \%$ & Rare
\end{tabular}

\section{Clinical features}

1. GI Bleeding - The most important, dreaded and dramatic presentation of PHT is GI bleeding and is the commonest reason for patients to visit a hospital. Bleeding is spontaneous, profuse, and painless. Most of the time bleeding is from esophageal varices. In about $2-10 \%$ cases the bleeding may be from gastric varices. In cirrhotics about

E-mail address: jkbanerjee4676@yahoo.co.in. 0377-1237/\$ - see front matter @ 2012, Armed Forces Medical Services (AFMS). All rights reserved. doi:10.1016/j.mjafi.2012.04.008 
$30 \%$ patients with varices will bleed. Generally bleeding stops spontaneously in 50\% patients. There are about $70 \%$ chances of rebleed within a year. About $20-30 \%$ patients are likely to die in each episode of bleeding. Risk of death is higher in cirrhotics especially with poor liver function, like in Child's Group C where the risk of death is about $70 \%{ }^{1}$

2. Splenomegaly - is present in all cases of PHT. The splenic enlargement is maximal in cases of NCPF. The cirrhotics may have only minimal enlargement of spleen with PHT. The spleen may regress in size after a recent bleed. These patients may have associated hypersplenism and therefore may require surgical intervention. Mild hypersplenism as manifested by thrombocytopenia and leucopenia is seen in $40-80 \%$ of patients and may require splenectomy. ${ }^{3,4}$

3. Ascites - indicates hepatic decompensation. A prominent feature in HVOTO, it is rarely seen in patients with EHPVO but may be seen in about $10 \%$ cases of NCPF. ${ }^{3}$ Presence of ascites also makes interventional investigations like a liver biopsy - a dangerous affair as the tamponade action of abdominal wall after the puncture of liver is not possible in presence of ascites.

4. Portosystemic encephalopathy - is defined as an abnormality of brain function due to disease of liver and is a sign of liver decompensation. Early symptoms of encephalopathy are subtle but can be identified by bedside tests like changes in hand writing, inability to copy a five pointed star, Reitan number connection test etc. ${ }^{1}$ As the severity of encephalopathy increases, patient passes into deep coma.

5. Signs of liver failure - may be apparent in cirrhotics like palmar erythema, gynecomastia, spider naevi and loss of axillary and pubic hair.

\section{Investigations}

Investigations will depend upon the situation in which patient is seen. They are grouped under the following situations:

1. In an elective situation

a. Hematology - especially to look for any evidence of hypersplenism.

b. Liver function tests - to differentiate cirrhotic from non cirrhotic portal hypertension and to classify them as per Child - Pugh classification

c. Coagulation profile

d. Viral markers

e. Liver biopsy - especially to ascertain the etiology of cirrhosis. For this patient should not have ascites and should have corrected coagulation parameters.

f. Upper GI endoscopy - for documentation and grading of esophageal varices, gastric varices, any PHT gastropathy and any other cause of hematemesis.

g. USG - is done to demonstrate collaterals in splenic hilum and elsewhere, to see the echotexture of liver, to look for any ascites and also to assess splenic vein and left renal vein as a pre operative evaluation.

h. Spiral CT angiography - gives a good picture of portal venous system and collaterals. This information is useful if a surgical procedure is being contemplated. i. MR Angiography - gives similar information as CT, however it delineates intravenous thrombosis better hence this investigation is very useful in a condition like Budd - Chiari syndrome.

2. In an emergency situation

Clinical examination for splenomegaly is sometimes more than enough in a situation of massive GI bleeding, to diagnose PHT.

a. Upper GI endoscopy is required more as a therapeutic procedure than a diagnostic procedure in such conditions.

b. Liver function tests are required to assess condition of liver to differentiate from cirrhotics

c. USG abdomen as a quick bedside assessment of liver and portal venous system is quite informative and should be done.

\section{Management}

Mere presence of PHT is not an indication for an active treatment other than simple supportive measures. Main indication of an operation is GI bleeding. Rarely surgery may be done for hypersplenism, intractable ascites ${ }^{5}$ or portal biliopathy. ${ }^{3}$ There is no role of a prophylactic operation for PHT to prevent bleeding in patients with cirrhosis or non cirrhotic portal fibrosis as it has not been shown to be of any survival benefit and the patients have a very high incidence of morbidity due to encephalopathy.

\section{Prophylactic operation}

Prophylactic operation is considered to be of benefit in only those patients who have a normal liver function hence is recommended only in extra hepatic portal venous obstruction. This is especially true for the poor patients of tropics where majority of patients of EHPVO are from poor socio economic background, are located in far flung areas away from specialized centers, cannot afford repeated endoscopic treatment for obliteration of esophageal varices and generally take a diet with minimal amount of animal protein further reducing the risk of portosystemic shunt encephalopathy. Hence a one - time prophylactic shunt may reduce the risk of their dying from GI bleed before they can reach a specialized center. $^{6}$

\section{Operation in bleeding patients}

a. Emergency procedures

i. Establish diagnosis

ii. Resuscitation and supportive measures including blood transfusion

iii. Pharmacological agents for variceal bleeding like beta blockers, nitrates etc, vasopressin, terlipressin, hormonal agents like somatostatin, octreotide etc

iv. Endoscopic therapy like endoscopic sclerotherapy (EST) or endoscopic variceal ligation (EVL) are very effective and are able to control acute variceal bleeding in about $90 \%$ of cases. Rebleed may occur in about $9-33 \%$ of cases 
which are either self limiting or easily controllable endoscopically. ${ }^{3}$

v. Role of esophageal balloon tamponade is fading away in view of wide spread availability of endoscopic facilities. However it still has a role in remote areas where the patient needs stoppage of acute bleeding and exsanguation before reaching a center with endoscopic facility.

vi. Transjugular intrahepatic porta systemic shunt (TIPS) if available is a useful bridging procedure to liver transplantation in patients with poor liver function and acute variceal bleeding by reducing the portal venous pressure. The procedure is however costly and has limited availability. Rate of shunt blockage is also high.

Emergency non shunt surgical procedures

Emergency non shunt surgical procedure of choice is Sugiura's devascularisation - transabdominal gastroesophageal devascularisation with gastroesophageal stapling.

\section{Emergency portosystemic shunt}

Though this procedure was initially tried in all types of PHT, however it was soon given up for cirrhotics in view of high (about 50\%) mortality in these cases due to post op liver failure. In NCPF and EHPVO the post op mortality was around $10-18 \%$ and rebleed rate was much less than devascularisation procedures. ${ }^{7,8}$

\section{b. Elective management}

i) Non operative methods: as described above in the emergency management

ii) Elective operations

a. Shunts

b. Non shunt operations

\begin{tabular}{lll} 
Shunts & \multicolumn{1}{c}{ Non shunt operations } \\
\hline Total & $\begin{array}{l}\text { Portocaval } \\
\text { Lienorenal }\end{array}$ & $\begin{array}{l}\text { Gastroesophageal devascularisation } \\
\text { with or without esophageal }\end{array}$ \\
& $\begin{array}{l}\text { Mesentericocaval } \\
\text { Mesentericoarterial }\end{array}$ & transaction \\
Selective & $\begin{array}{l}\text { Distal lienorenal (DSR) } \\
\text { Coronary Caval }\end{array}$ & Loop interposition - colon \\
& or jejunum
\end{tabular}

\section{Merits and demerits of various commonly done operations}

(a) Portocaval shunt: is an easier operation than other shunt surgery but cannot be done in EHPVO as requirement is of a patent portal vein. In end to side portocaval shunt, due to shunting of total portal venous blood there is $40 \%$ incidence of encephalopathy in cirrhotics and $20 \%$ incidence in NCPF. The incidence of encephalopathy is not low in side to side shunting due to shunting of hepatic arterial blood occurring as a result of backflow in to IVC

(b) Proximal lienorenal shunt: is probably the most useful shunt in patients of tropics as majority of them are EHPVO with some element of hypersplenism and having a good liver function. The proximal shunt includes splenectomy which cures hypersplenism too. These shunts are however difficult to perform but remain patent in about $90-95 \%$ in long term follow up. ${ }^{9-12}$

(c) Distal lienorenal shunt (DSRS): initially these shunts are selective, hence cause much less encephalopathy. However all these develop reanastomosis between the disconnected veins and at the end of two years have no difference in encephalopathy or length of survival between these and total shunts.

(d) Mesocaval shunt: done with a $16 \mathrm{~mm}$ Dacron graft is easier to perform but have a very high incidence of shunt occlusion

(e) Gastroesophageal devascularisation: achieves disconnection between all the systemic and portal venous system around the lower end of esophagus thus stopping the bleeding from varices. Though the initial control of bleeding is good and encephalopathy rate is low but this has a very high rebleed rate of about $30-40 \%$.

\section{Results of operation}

(a) Operative mortality varies between 15 and 90\% depending upon the liver function. In Child's A it may be as low as $15 \%$ but in Child's C, it may be as high as $90 \%$. In NCPF and EHPVO the mortality is very low in elective situation.

(b) Rebleeding rate is about $10 \%$ after a lienorenal shunt but is very high to the extent of $30-40 \%$ after a devascularisation procedure. Rebleeding in shunt surgery is directly proportional to shunt occlusion rate. In case of rebleed after a shunt operation, endoscopic management of the varices is probably the best alternative.

\section{Long term survival}

As majority of patients in tropics are of EHPVO, long term survival of these patients is significantly better after a shunt surgery than non operated patients. Though many recent studies have shown almost comparable rates of control of acute bleed, rebleed and overall long term survival with endoscopic variceal sclerotherapy, ${ }^{13}$ yet in a country like India, where expert endoscopic therapy and blood banking facilities in many remote areas are still sub optimal, a onetime surgical procedure in the form of a splenectomy and portosystemic shunt, in those with good liver function like EHPVO or NCPF is still a better option. Moreover, the control of portal hypertensive gastropathy, portal biliopathy and growth retardation in these patients can be addressed only by a portosystemic shunt. ${ }^{2,8}$ The average survival of cirrhotic patients after shunt surgery, however, is only 5 years and a liver transplantation is the only definitive mode of treatment in these patients.

\section{Conclusion}

(a) The etiology of PHT varies significantly between the patients of Western countries and those in tropical countries. In tropical regions, majority are either non cirrhotic 
portal fibrosis (NCPF) or extra hepatic portal venous obstruction (EHPVO) with good liver function.

(b) Massive splenomegaly and hypersplenism is usually an important part of the presentation and merit treatment on their own.

(c) Most important complication of PHT is variceal bleeding and this needs prompt treatment in a specialized center. All non surgical methods, pharmacological methods and endoscopic measures should be tried, however, if necessary to control bleeding surgical methods may also be tried

(d) An emergency shunt procedure is probably the best surgical method in extra hepatic obstruction with PHT. However, in a cirrhotic with poor liver function surgery has high mortality, results in high incidence of encephalopathy and liver failure. In these patients a gastroesophageal devascularisation procedure has lesser complications though has high rebleed rate. TIPS, if available, is a good option.

(e) An elective shunt procedure in EHPVO improves survival significantly though it may not be so in cases of cirrhotics, especially those with poor liver function.

\section{Conflicts of interest}

None identified.

\section{R E F E R E N C E S}

1. Mathur SK. Cirrhosis and portal hypertension. In: Haribhakti S, ed. Clinical GI surgery - a reference book for surgeons. 1st ed. Ahmedabad: Haribhakti Education Foundation; 2008:835-856.
2. Pande GK, Reddy VM, Kar P. Operation for portal hypertension due to extrahepatic obstruction: results and 10 years follow up. Br Med J (Clin Res Ed). 1987;295:1115-1117.

3. Sarin SK, Agarwal SR. Extrahepatic portal venous obstruction. Semin Liver Dis. 2002 Feb;22(1):43-58.

4. Mitra SK, Kumar V, Dutta DV. Extrahepatic portal hypertension: a review of 70 cases. J Pediatr Surg. 1978;13:51-54.

5. Nundy S. Portal hypertension. In: Srivastava SK, ed. Modern concepts in surgery. 1st ed. New Delhi: Tata McGraw Hill; 1992:142-152.

6. Pande G, Sahni P, Nundy S. Extrahepatic obstruction causing portal hypertension. J Gastroenterol Hepatol. 1988;3:99-107.

7. Chaudhary A, Aranya RC. Devascularisation following endoscopic sclerotherapy of esophageal varices: dangers and difficulties. Br J Surg. 1991 Oct;78(10):1249-1251.

8. Prasad AS, Gupta S, Kohli V, Pande GK, Sahni P, Nundy S. Proximal splenorenal shunt for extra hepatic portal venous obstruction in children. Ann Surg. 1994 Feb;219(2):193-196.

9. Orloff MJ, Orloff MS, Girard B, Orloff SL. Bleeding esophagogastric varices from extrahepatic portal hypertension: 40 years experience with portal-systemic shunt. J Am Coll Surg. 2002;194:717-728.

10. Bismuth H, Franco D, Alagille D. Portal diversion for portal hypertension for children: the first ninety patients. Ann Surg. 1980;192:18-24.

11. Alvarez F, Bernard O, Brunelle F, Hadchouel P, Odievre M, Alagille D. Portal obstruction in children.II. Results of surgical portosystemic shunt. J Pediatr. 1983;103:703-707.

12. Orloff MJ, Orloff MS, Rambotti M. Treatment of bleeding esophagogastric varices due to extra hepatic portal hypertension: results of portosystemic shunts during 35 years. J Paediatr Surg. 1994 Feb;29(2):142-151. discussion 151-154.

13. Thomas V, Jose T, Kumar S. Natural history of bleeding after esophageal variceal eradication in patients with extrahepatic portal venous obstruction: a twenty year follow up. Indian $J$ Gastroenterol. 2009;28:206-211. 